(C) 2019. This manuscript version is made available under the CC-BY-NC-ND 4.0 license http://creativecommons.org/licenses/by-nc-nd/4.0/

Safety Science, 117, 490-500. https://doi.org/10.1016/..ssci.2016.10.019

\title{
Delivering high reliability in maternity care: in situ simulation as a source of organisational resilience
}

\author{
CARL MACRAE \\ Department of Experimental Psychology \\ University of Oxford \\ Tinbergen Building, 9 South Parks Road \\ Oxford OX1 3UD \\ carlmacrae@mac.com
}

TIM DRAYCOTT

School of Social and Community Medicine

University of Bristol

Southmead Hospital

Bristol BS10 5NB

tim.draycott@bristol.ac.uk 
Keywords: simulation; resilience; high reliability; emergencies; patient safety 


\section{Delivering high reliability in maternity care: in situ simulation as a source of organisational resilience}

The fields of resilience engineering and high reliability organising both seek to explain the key sources and characteristics of safety in organisations that operate under conditions of considerable complexity, variability and surprise. A key focus in both of these fields is explaining how organisations can use adaptive and flexible work processes to deliver safe and reliable services, and how organisations can draw on past events and new experiences to increase their capacity to handle disruptive and unexpected events. To explore these issue, this paper develops an analysis of the routine use of on-site or 'in situ' simulation of emergency events as part of a systematic approach to safety management in the healthcare setting of maternity care. This analysis identifies three core organising processes through which in situ simulation can act as a source of organisational safety: relational rehearsal, system structuring and practice elaboration. We use this analysis to examine the opportunities that exist to develop more integrated explanatory accounts of high reliability organizing and resilience engineering. 


\section{Highlights}

- Routine use of on-site or 'in situ' simulation is a driver systemic safety

- In situ simulation supports both adaptive change and stabilizing routinisation

- In situ simulation creates symbolic disruptions that lead to material adaptation

- In situ simulation allows work-as-imagined to be tested against work-asdone

- In situ simulation both enhances positive practices and addresses weaknesses 


\section{Introduction}

One of the most pressing challenges facing modern organisations is the need to deliver safe and reliable services under conditions of considerable complexity, change and surprise. Understanding the key sources and characteristics of safety in complex organisational settings is therefore a significant priority in both research and practice, and has become the focus of two broad fields of research. The field of high reliability organising is largely focused on the role of cognitive and organisational processes that allow unexpected and out-of-ordinary events to be reliably detected and flexibly responded to before they escalate out of control (e.g. Roberts, 1990; Weick, Sutcliffe and Obstfeld, 1999). The emerging field of resilience engineering (e.g. Hollnagel, Woods and Leveson, 2006; Hollnagel, Paries, Woods and Wreathall, 2013) encompasses a broad set of ideas, but is primarily focused on understanding how ongoing adaptations, adjustments and variations in organisational practice can underpin organisational safety. These two fields of inquiry remain largely disconnected despite their apparently shared aim of explaining how organisations can deliver safe and dependable services through adaptive and flexible work processes.

This disconnect points to important fundamental questions that remain in our understanding of organisational safety. These questions largely concern the tensions between organisational stability and change (Wears, 2015; Weick, Sutcliffe and Obstfeld, 1999; Macrae, 2010; 2013). How can organisations deliver 
services that are standardised, replicable and predictable-while embracing the variations, disruptions and unexpected events that are inherent to organisational life? And how can organisations sustain stable activities and reliable routineswhile continually adapting and improving their activities in light of past experience, current knowledge and new evidence? To address these questions, in this paper we analyse the routine use of on-site or 'in situ' simulation of emergency events as part of a systematic approach to managing and improving safety in maternity care. We examine how the regular in situ simulation of unpredictable emergency events can simultaneously act as a source of organisational stability and organisational adaptation. We use this analysis to explore the nature of organisational safety, and examine the opportunities that exist to develop more integrated explanatory accounts of organisational high reliability and resilience.

This paper is structured as follows. First, we introduce simulation as a safety improvement strategy and explain why healthcare in general, and maternity care in particular, offer an especially productive arena in which to explore the interconnections between organisational resilience and high reliability. Next, we describe the particular challenges of maternity safety, along with a well established maternity safety improvement programme that has in situ simulation as its core. Then, an analytical account is developed that aims to characterise how the routine use of in situ simulation can act as a systemic source of safety, through three organisational processes: relational rehearsal, system structuring and 
practice elaboration. After that, we explore the implications for theories of high reliability organising and resilience engineering, and propose two underpinning organisational mechanisms that point to how we might better understand the nature of organisational safety and connect these fields.

\section{Emergencies, simulation and system safety}

Handling unexpected and unpredictable emergency situations is a challenge faced in many safety-critical organisations and is a key concern that connects the fields of high reliability organising and resilience engineering. Emergency situations are-by their nature-surprising, unusual and rare events in which people must manage demanding, time-pressured, volatile and highly hazardous situations that pose considerable threats to safety. Due to the high stakes involved and the infrequency of occurrence, many safety-critical settings practice and prepare for emergencies through the use of simulated emergency scenarios (Weick, 1987; LaPorte, 1994; Dekker et al, 2008). Simulation provides a way of recreating different elements of emergency situations so as to represent or amplify 'realworld' experiences (Gaba, 2004). The impact of simulation varies, and can be influenced by a range of factors including how well simulations correspond to actual events encountered in daily practice, the authenticity of simulated scenarios and settings, and how fully participants engage in simulated exercises (eg Haji et 
al, 2016; Crofts et al, 2006; Dutta et al, 2006). At core, simulation as a safety strategy embodies many of the key commitments of high reliability organising and resilience engineering: it is deeply concerned with the particulars of practical work, it is focused on developing the positive attributes and active capacities that underpin safe performance, and it is oriented to improving safety through recognising and responding to disruptions.

Healthcare has a long tradition of using formalised simulation training to improve patient safety that stretches back several decades (Rosen, 2014; Schmidt et al, 2013), including simulating surgical complications (Arora et al 2015; Gaba et al 2001), anaesthesia crises (Gaba et al, 2014; Goldhaber and Howard, 2013), emergency medicine (Wisborg et al, 2006) and obstetric emergencies (Ayres-deCampos et al, 2011). Healthcare simulation has primarily focused on developing the skills and knowledge of individuals and the capacities of teams to communicate and work together effectively (Tan and Sarker, 2011; Gaba, 2010), and simulations are often conducted in specially designed standalone simulation centres. More recently, the benefits of using on-site or in situ simulation to test and probe real-world organisational systems have begun to be more widely recognised (Fent et al, 2015; Auerbach et al, 2015; Walker et al, 2013).

In situ simulations are conducted in the actual settings in which people routinely work, and typically bring together the wide variety of professionals involved in managing particular types of healthcare emergency. This allows the 
skills of individuals and multidisciplinary teams to be developed and optimised. It also allows for broader, more systemic activities of safety improvement to be undertaken. In particular, in situ simulation allows deficiencies in processes, equipment and organisational systems to be identified and improved proactively, before an actual emergency situation or adverse event involving a real patient occurs (Patterson et al, 2013; Hammam et al, 2009; Ventre et al, 2014; Riley et al, 2010). However, despite the increasingly widespread use of in situ simulation in different settings, our understanding of the mechanisms through which in situ simulation can increase the reliability and resilience of organisational activities remains under-developed (Driver et al, 2011).

\section{Obstetric emergencies and in situ simulation}

One of the most successful long-term applications of in situ simulation as part of a systemic patient safety improvement programme has been developed in the healthcare setting of maternity care. Maternity care offers an exemplary setting to examine how services can be delivered reliably and safely in the face of complexity, variation and surprise. Maternity care-like much of healthcareinvolves the coordination of a diverse range of professionals who must routinely work together to manage a wide variety of patients, conditions and interventions. Scientific evidence, medical technology and clinical practice evolve rapidly. And in 
maternity care in particular, an extremely wide spectrum of care is delivered. Childbirth is a natural process but it brings with it a range of risks, and the transition from a normal, routine delivery with little or no intervention to an urgent and life-threatening obstetric emergency can happen rapidly and with little warning. Around one in twelve deliveries are associated with some form of adverse event (Nielson et al, 2007). Preventable harm can be catastrophic to the women, children and families involved (Kirkup, 2015). In addition to these deeply personal tragedies, preventable harm related to childbirth is also extraordinarily expensive: substandard maternity care and its consequences cost the National Health Service in England £3.1 billion between 2000-2010 (NHSLA, 2013).

Obstetric emergencies take a variety of forms and are rare events but demand rapid, reliable and complex management. For example, each year a typical maternity unit in the UK will be required to manage only two cases of eclampsia: a serious and life threatening condition related to high blood pressure in the mother that involves convulsions and can lead to coma (Thompson, Neal and Clark, 2004). Like all obstetric emergencies, eclampsia requires immediate and complex emergency management involving a range of clinical interventions delivered by a variety of professionals. Managing eclampsia requires control of the mother's airway, delivery of oxygen, administration of magnesium therapy to stop seizures, control of hypertension, electronic monitoring of the baby, testing of the mother's blood and other physiological signs, as well as delivery of the baby (Thompson, Neal and Clark, 2004). This emergency response requires the effective and rapid 
coordination of midwives, obstetricians, anaesthetists, healthcare support workers, laboratory staff, porters, switchboard operators and also operating theatre staff if a caesarian section is required-along with all of the associated equipment, materials and supplies.

Other rare but serious obstetric emergencies include, for example: cord prolapse, where the umbilical cord is delivered before the baby, necessitating emergency intervention and urgent caesarian section; post partum haemorrhage, where serious bleeding occurs soon after birth requiring rapid diagnosis and treatment; shoulder dystocia, where the baby's shoulder gets stuck during delivery, requiring careful intervention to prevent serious damage to the baby's tissues and nerves which can lead to permanent injury and paralysis; and maternal collapse, where the mother's health rapidly and severely deteriorates, and may include heart attack and loss of consciousness.

\subsection{Practical Obstetric Multi-Professional Training}

An increasingly widespread approach to improving safety in maternity care is the use of various methods of emergency simulations and 'drills' targeted at improving the handling of obstetric emergencies. (RCM, 2002; RCOG, 1999; TJC, 2004, NHSLA, 2000; MCHRC, 2000). One of the most established and successful 
applications of in situ simulation in maternity care is the Practical Obstetric Multi-Professional Training (PROMPT) programme. PROMPT is an integrated and systematic approach to safety improvement in maternity care that encompasses different forms of skills training, emergency simulation and systems improvement (Winter et al, 2012a). The PROMPT programme has been developed over fifteen years at Southmead hospital in the South West of the United Kingdom. PROMPT has subsequently been adopted by around $85 \%$ of UK units and is increasingly being used internationally, including in the US, Australia, Asia and Africa (Draycott, 2013). At its core, PROMPT represents an integrated approach to safety improvement that is supported by a set of practical manuals, simple tools and systems design processes, all of which are put into practice and regularly tested and improved through the routine use of in situ simulations of obstetric emergencies. These simulations bring together multi-professional clinical teams in their normal working environment to rehearse, reflect and improve on their collective practice and the systems, processes and tools that support this practice. The focus is therefore both on simulating and improving skills, and simulating and improving systems (Box 1).

Since the development and introduction of PROMPT in 2002, the maternity unit at Southmead has demonstrated significantly improved safety outcomes, including a 70\% reduction in neonatal injuries related to situations in which the baby's shoulder becomes obstructed during labour (brachial plexus injuries related to shoulder dystocia); a 50\% reduction in infants born with low measures 
of physical condition (five minute Apgar scores of less than seven); and significant reductions in the time between the diagnosis and delivery of babies in emergency situations where the umbilical cord is delivered before the baby (cord prolapse), improving outcomes for babies (Draycott et al, 2006; Draycott et al, 2008; Siassakos et al, 2009; Siassakos et al, 2011). Over this same period, maternity related litigation claims at the hospital have fallen from $£ 25 \mathrm{~m}$ to $£ 3 \mathrm{~m}$ (Draycott et al, 2015). The implementation of PROMPT has also been associated with positive outcomes for staff, with staff attitude surveys indicating a positive safety culture, excellent teamwork climate and high job satisfaction (Siassakos, 2011). Achieving such tangible improvements in safety outcomes is unusual in healthcare, and places Southmead as one of the safest maternity units in the world in terms of birth complications (Draycott, 2013). This success is particularly striking given that other programmes that have aimed to improve skills and knowledge in relation to obstetric emergencies have, in some cases, ended up increasing the rate of injuries and adverse outcomes (MacKenzie et al, 2007; Draycott et al, 2015).

The PROMPT programme therefore offers a particularly valuable opportunity to explore and characterise the use of what might be termed systemic in situ simulation: where the simulation of emergency events and crises is used to routinely probe, test and improve organisational systems and situated practices as part of a long-term, systematic and structured approach to safety improvement. In this sense, rather like the foundational studies of high reliability organising, 
PROMPT is an exemplary case of how in situ simulation is currently "working in practice but not in theory" (La Porte, 1991). PROMPT represents a demonstrably successful suite of safety improvement activities that have been developed over many years, drawing on deep practical and contextual knowledge (e.g. Siassakos et al, 2013; Siassakos et al, 2009; Draycott et al, 2015). Accordingly, the purpose of this paper is to reflect on PROMPT as an exemplary case, using it to characterise the key organising processes through which in situ simulation can underpin organisational resilience and high reliability-and to then use that analysis to explore connections between these two fields. The characterisation developed here thus represents a moment in an ongoing theorising process (Weick, 1995), and offers an emerging account of some of the key reliabilityseeking and resilience-enhancing characteristics of systemic in situ simulation.

\section{Box 1. In situ simulation in PROMPT}

Core features of the Practical Obstetric Multi-Professional Training programme at Southmead Hospital include:

- Training and simulation day held once every 8 weeks including a range of emergency drills that simulate different obstetric emergency scenarios.

- Simulations typically run in situ and involve the full multi-professional team, in the labour ward or typical place of occurrence for the simulated emergency.

- All staff must attend at least once a year, including midwives, obstetricians, anaesthetists and midwife care assistants. Annual attendance closely approximates 100\% (Siassokos 2009).

- Patient actors play the role of the mother, along with high-fidelity simulation equipment that mimics birthing scenarios and signs and 


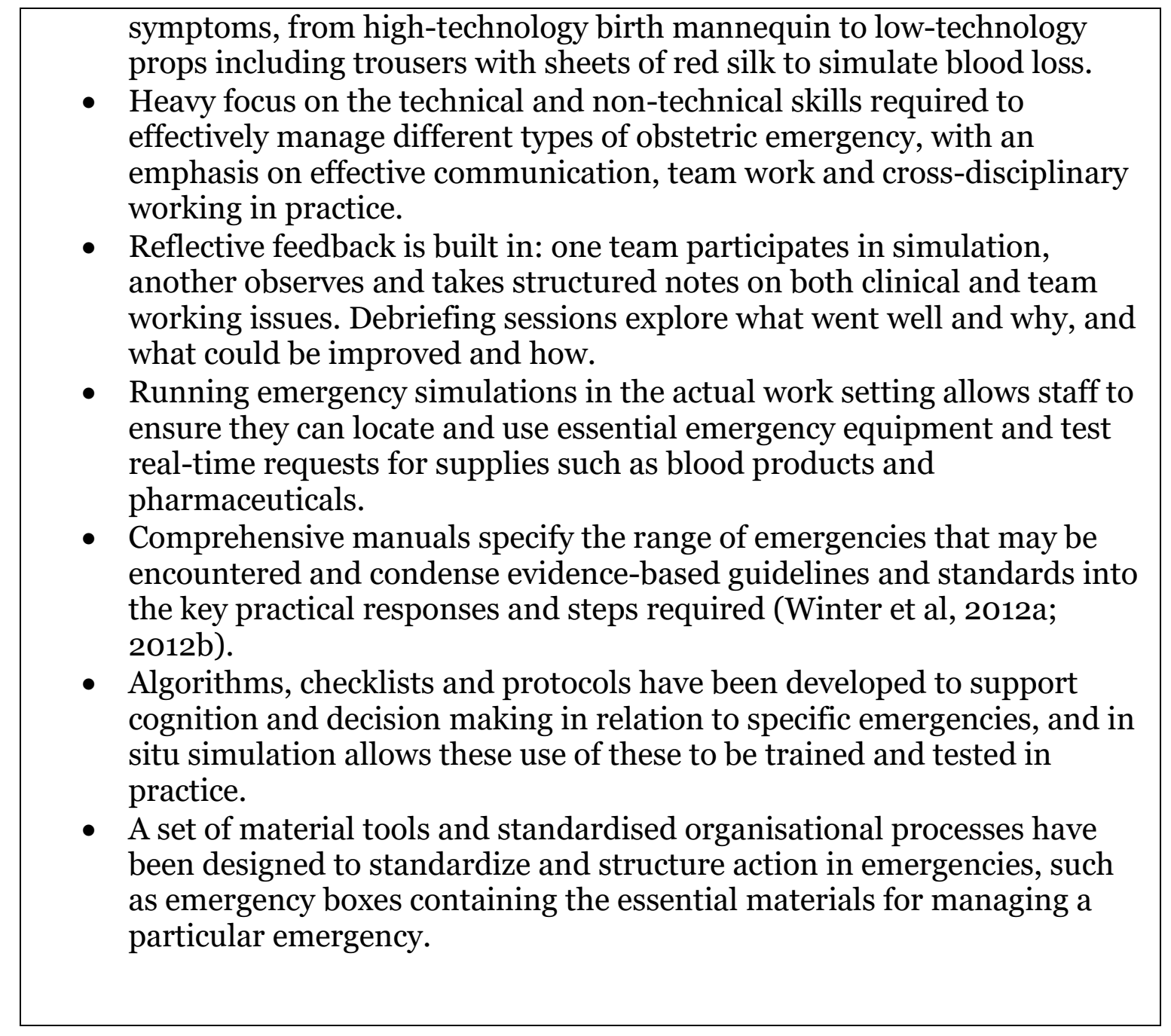

\section{The simulated sources of organisational safety}

Achieving high levels of safety in maternity care represents a considerable organisational accomplishment. It depends on maintaining an ongoing capacity to identify and manage rare obstetric emergencies while performing the normal, routine work of maternity care. Obstetric emergencies can develop rapidly and the 
outcome for both mother and baby depends on efficiently organising a complex set of activities. Maternity staff must be able to recognise and respond to obstetric emergencies swiftly, reliably coordinating complex emergency interventions by a diverse team of specialists. These emergency actions must be attuned to the specific situation at hand, responding to the unique signs, condition and history of the mother and baby, while ensuring that appropriate evidence based standards of care are delivered efficiently and compassionately. To support this, the material resources, clinical knowledge, practical skills and work environment must all be maintained and ready to support action at a moment's notice.

Our analysis here centres on identifying the organising processes and social factors through which in situ simulation acts as a source of organizational safety in maternity care. We identify three core processes that are supported by in situ simulation and that underpin high reliability: relational rehearsal, systems structuring and practice elaboration (Table 1). Relational rehearsal represents the social processes that are involved in building shared expectations, establishing patterns of collective working and maintaining trust amongst the many diverse professionals who must rapidly come together to respond to an obstetric emergency. System structuring concerns the processes that are involved in designing, testing and improving the organisational systems and infrastructures that support rapid and adaptive responses to emergency situations. Practice elaboration concerns the processes involved in examining, refining, improving and embedding clinical practices that allow timely and effective responses to a 
wide variety of problematic situations. In the sections that follow, each of these is examined in turn.

Table 1. The organizational mechanisms supported by systemic in situ simulation that contribute to organizational safety.

\begin{tabular}{|c|l|l|}
\hline Relational rehearsal & System structuring & Practice elaboration \\
\hline Shared expectations & Organisational improvement & Embodied wisdom \\
\hline Collective trust & Cognitive infrastructure & Reflective inquiry \\
\hline
\end{tabular}

\subsection{Relational rehearsal}

Social relations are the basis of resilience and high reliability (Weick and Roberts 1993). In situ simulation, as employed within the PROMPT system, provides a space and a set of activities that aim to establish social roles and relations that enable the rapid detection, communication and management of emerging 
problems and emergency events. These processes of relational rehearsal involve at least two core and interdependent elements: shared expectations and collective trust.

\subsection{1. $\quad$ Shared expectations}

In situ simulation allows the development of shared expectations regarding individual roles and responsibilities, the patterns of communication, and the flow of coordinated activity required to manage emergencies. Maintaining clear role structures in high performing teams is essential to support ongoing understanding and coordination (Weick, 1993). The safe management of obstetric emergencies requires rapid and coordinated action from a range of different professionals. This emergency work is critically important but relatively unusual. That is, the work that is most consequential to safe outcomes is also the most complex and the least routinely experienced, and is performed by teams who may not have worked together in similar circumstances. Engaging in simulated emergencies allows individuals to understand the nature of their role in relation to the work of others during emergency situations, creating shared expectations regarding the scope and remit of each role within a rapidly formed team. A central feature of high reliability organising is the appreciation that each individual has of their place in a broader system of activity, and a shared understanding and heedfulness towards 
the actions of others (Weick and Roberts, 1993). In situ simulation also allows these shared expectations to be formed across broad systems of activity, including team members who may be present at the bedside but also others elsewhere in the organization, such as blood bank staff or paramedics. In situ simulation builds these practical expectations of how specific roles interface with the tasks of others. These shared expectations can then provide a normative benchmark against which deviations can be detected (Rochlin, 1989; Weick and Roberts, 1993; Resar, 2006).

\subsection{2. $\quad$ Collective trust}

Working together in simulated emergencies allows individuals to build confidence in their own abilities and trust in the ability of colleagues to act effectively. Communication barriers and professional silos can be a considerable challenge in safety-critical settings, and inter-professional barriers have contributed to serious safety issues in healthcare -and maternity disasters in particular (Kirkup, 2015). In situ simulation helps to establish an open and safe space where professionals from different backgrounds can work on developing their strengths, acknowledge and address their weaknesses and build confidence in their collective capabilities. Building trust is aided by the design of the simulation events: PROMPT is not a test of individual performance (Weinger et al, 2014; Winter et al, 2012b) but 
provides a space to reflect on and improve practices, to share knowledge and practical experience, and to strengthen social and professional bonds. Fun is an important feature: simulations should be challenging and taken seriously, but also enjoyable (e.g. Gallagher and Corrado, 2014) and lighter hearted moments are incorporate into PROMPT events to increase open interactions. More subtly, PROMPT's in situ simulations emphasise the role of the systems in which people work-and how the actions of individuals helps create and constitute the system within which everyone works. Building a shared understanding of the role of local context and wider systems in individual and team performance reduces tendencies to blame individuals (Sutcliffe and Weick, 2003), and helps to build collective trust. In combination, all of this can reduce fear: fear of speaking up, fear that others will not perform as needed, and fear that you yourself do not know how to respond when the worst happens.

\subsection{System structuring}

Some of the most fundamental functions of in situ simulation, as employed within the PROMPT system, are the design and improvement of the organizational systems that support the management of obstetric emergencies. System structuring consists of activities that are targeted at designing the sociotechnical processes of maternity care, and testing and refining those designs in the practical 
settings in which they are enacted. These activities can be characterised as primarily focusing on two key elements: organisational improvement and cognitive infrastructure.

\subsubsection{Organisational improvement}

In situ simulation provides a space in which idealised models of organisational activity can be tested, adapted and implemented in practice. And it also provides a space in which deficiencies in current work systems and organisational arrangements can be identified and addressed. This allows 'systems probing' (Driver et al, 2011) for organizational improvement: testing and adapting the local contexts, materials, resources and organisational processes that support practical work-essential but mundane practicalities such as ensuring that equipment is accessible and functional, and that people know how to use it. One particularly striking example of organisational improvement in the PROMPT programme is the development and use of 'emergency boxes'. Emergency boxes-such as the 'Post Partum Haemorrhage (PPH) box' or the 'Eclampsia box' (Draycott, Broad and Chidley, 2000) - contain the common materials and tools that are required to handle a specific emergency situation. Emergency boxes represent a simple organisational design for reliably providing the right equipment at the right timebut the box, on its own, achieves little. In situ simulation allows the practical use 
of emergency boxes to be tested, routinized and improved, and in so doing, allows reliable social and organizational processes to be developed around the implementation and use of standardised tools and protocols.

In situ simulation also allows the broader organisational systems that surround emergency response to be routinely tested and improved. In the case of a post partum haemorrhage, these include the emergency call system to summon assistance, and the need for rapid delivery of specific blood products. Each of these in itself represents a complex sociotechnical process. For example, an emergency buzzer system consists of the technical alarm that must be maintained, serviceable, accessible and audible, a 'code red' protocol and pre-determined list of who to contact and who should attend for different scenarios and situations, along with all the social arrangements and expectations required to support this. Regularly testing these systems allows for deficiencies to be identified and practical improvements to be made. More fundamentally, for staff, the experience of regularly testing and improving systems can foster a more generalised intolerance of glitches in systems and mis-specified processes and can help build a social license for adapting work systems. For example, instituting the routine of hanging a sign on the door during team debriefings that indicates who on the ward has the keys to the drug cabinet, to avoid unnecessary and repeated interruptions. These are all key drivers of high reliability organising (Schulman, 2004; Roberts and Creed, 1993). 


\subsubsection{Cognitive infrastructure}

Safe systems of collaborative work depend on maintaining situational awareness, shared understanding and heedful interaction in relation to risk (Weick and Roberts, 1993). This cognitive infrastructure of high reliability (Weick, Sutcliffe and Obstfeld, 1999) can in part be supported by standardized cognitive aids such as checklists, protocols, decision algorithms and emergency manuals that facilitate coordinated thinking and acting (Marshall, 2013). PROMPT is based on a variety of cognitive aids that condense a huge amount of established knowledge, and hundreds of pages of clinical evidence and guidelines, into easily accessed tools that orient attention and structure action (Draycott, 2006). Like many cognitive aids, these tools capture essential-and often easily overlooked-practical actions, such as checking that the date and time on cardiotocography (CTG) machines are always set and checked (Winter et al, 2012a). These tools, in the form of decision algorithms and itemized checklists, provide anchors around which coordinated activity can unfold. They constitute representational media that can share the cognitive load in pressured situations, create shared mental models of normative and actual activities, support distributed cognition (Hutchins, 1996) and help to build operational representations (Bigley and Roberts, 2001) that capture current operational status and structure key decision points. To do this effectively, cognitive aids must be available, meaningful and actively integrated into ongoing 
activities (Goldhaber-Fiebert et al, 2016; Goldhaber-Fiebert and Howard, 2013, Marshall, 2013). In situ simulation provides a regular and protected space in which staff can familiarise themselves with the use of different cognitive aids in practice, and integrate these into their collective patterns of work. This also allows cognitive aids to be tested and improved through iterative practical feedback, along with other organizational processes.

\title{
4.3. Practice elaboration
}

\begin{abstract}
A key function of in situ simulation is the elaboration, refinement and improvement of professional practice. Practice elaboration represents a set of processes that support the rehearsal and refinement of work practices and the purposeful testing of new or unusual practices. When conducted in the local contexts and settings in which people normally work, in situ simulation allows the interaction between practices, contexts and organisational systems to be reflected on and refined. Practice elaboration can be characterised as focusing on two key elements: embodied wisdom and reflective inquiry.
\end{abstract}




\subsection{1. $\quad$ Embodied wisdom}

In situ simulation allows abstract knowledge to be connected with practical, embodied work in the settings in which that work is actually done. In maternity care, many of the key responses required in different types of emergency will be understood by healthcare professional in the abstract, through professional education and established guidelines (e.g. RCOG, 2011). However, this knowledge may be rarely drawn on in day-to-day work. In situ simulation allows that formal knowledge to be put into practice in the natural settings in which people work, developing understanding not only of what to do, but how to do it. Tacit and embodied knowledge of how to perform skilled clinical tasks is central to safe healthcare, but tacit knowledge can't be transferred or taught directly; it has to be developed by doing (Cook and Brown, 1999). PROMPT simulation sessions are particularly concerned with developing the particular technical skills required to manage obstetric emergencies. Importantly, this provides a space in which practical tips, recipes and heuristics can be shared and circulated amongst staffsuch as the 'Pringle tube' technique for positioning one's hand while safely manipulating a shoulder dystocia emergency (touch all fingertips and thumbtip together as if reaching into a Pringle tube) (Cornthwaite et al 2015), or the perceptual rules of thumb for estimating blood loss (a bed pan is around half a litre; blood spilling from the bed onto the floor is around 2 litres) (Winter et al, 2012a). Circulating and rehearsing this embodied knowledge is an important process of stabilizing and improving the practical work of clinical care. Equally, 
for each of the main types of obstetric emergency, the PROMPT sessions and associated manuals highlight the most common practical problems and difficulties that staff might encounter in real-world settings. Many of these challenges have been discovered through prior drills and simulations, and have themselves been woven back into the manuals and scenario plans (Winter et al, 2012a).

\subsection{2. $\quad$ Reflective inquiry}

Regular in situ simulation creates a space for routine reflection on, and observation of, practical work in the settings it is actually conducted in. Reflective inquiry involves turning work itself into an object of inquiry, allowing practices and the systems that surround them to be examined, reflected on, reorganised and improved (Miettinen and Virkkunen, 2005). Reflecting on the work, and on the way things actually get done, is an essential skill that can sometimes be regarded as beneath highly trained professionals in healthcare (Batalden, 2010). By turning this into a normalised and regularised way of working, regular in situ simulation allows the practices of reflective inquiry and adaptive change to be built into a broader system of safety improvement. PROMPT intentionally does not use in situ simulation as a test of individual knowledge and skills, but instead aims to create an environment in which professionals get used to observing, being observed and reflecting on their own work practices. Facilitated debriefs at the end of each 
simulated scenario provides an opportunity to reflect on performance in a collegiate environment, and to give and receive productive, constructive and improvement-focused feedback. This embeds reflective inquiry into the normal work environment, contributing to a culture in which noticing, speaking up and inquiring into practical problems is routine. More broadly, this helps to operationalise the principle that noticing is enhanced by acting: being empowered to inquire into and act on problems expands the range of problems that are noticed in the first place (Weick, Sutcliffe and Obstfeld, 1999). This can contribute to a culture in which people notice and act more-both in relation to positive features of practice to elaborate, and discrepancies in systems to address.

\section{Discussion: Rehearsing resilience and reorganising high reliability}

Analysing the routine use of in situ simulation in a maternity care setting offers a range of key insights regarding the organisational sources of safety, as well as the interconnections between theories of resilience engineering and high reliability organising. These insights focus on the nature of organisational stability and change, the relationship between proactive and reactive forms of safety management, and the interconnections between efforts to amplify and spread 
improvements and efforts to identify and address organisational weaknesses. One of the key areas of intersection revealed by our analysis of in situ simulation concerns the tension between stability and change as sources of organisational safety. Systemic in situ simulation can simultaneously act as both a source of standardisation and as a source of adaptation in organisational practices. In the short term, the rehearsal of emergency events allows the development of stable patterns of social interaction and allows essential elements of emergency response to be routinised. These routines and shared expectations can provide the foundation for more flexible and adaptive responses in moments of crises. Over the longer term, in situ simulation allows continual examination and adaptation of organisational activities. It provides mechanisms through which practical work and organisational systems can be reorganised and improved in light of past experience and new evidence. Systemic in situ simulation therefore sits at the intersection between stability and change, flexibility and standardisation, and routinisation and adaptation.

Another area of intersection is that in situ simulation provides a set of activities that simultaneously seek to amplify and develop the positive characteristics of systems and practices, while at the same time identifying and addressing weaknesses in those same systems and practices. In theory these processes are often distinguished as different modes of safety management, with the focus on one or the other: either amplifying 'good' practices, or addressing and mitigating 'bad' events. However, in practice it can be hard-if not impossible-to 
disentangle these two sources of organisational safety. The ways these processes intersect point to opportunities to refine our theories of safety, improvement and risk.

Finally, examining the systematic use of in situ simulation offers an exemplary case to explore how proactive and reactive activities of safety management are inextricably entwined. The timing of safety-when it occurs in relation to a disruptive event-has been a central concern in the fields of resilience engineering and high reliability organising. However, our analysis suggests that this tension can be resolved by shifting attention to the nature of the processes through which disruptive events can enact safety, rather than argue over their timing.

We discuss these interconnections between current thinking on resilience and high reliability by first analysing the complicated relationship between organisational strengths and organisational weaknesses in safety management. Then we explore the nature of proactive and reactive modes of safety. Finally we examine the deep and underlying tension between stability and change in organisational theories of safety. We use this analysis to point to new ways of connecting the fields of resilience engineering and high reliability organising. 


\subsection{Connecting strength and weakness}

A persistent debate in the theory and practice of organisational resilience and reliability is the appropriate balance between developing positive attributes of safety or addressing weaknesses-between the light and dark side of organisational safety. Historically, much of our knowledge of safety has been derived from moments of its absence: the analysis of accidents, incidents and failures (Reason, 2000; Macrae, 2014). Equally, many of the traditional strategies of safety and risk management have been oriented to episodes of weakness and failure. The definition of safety itself has often rested on a double negative: the avoidance of harm (Rochlin, 1999; Hollnagel, Wears and Braithwaite, 2015). This focus on the negative face of safety-on failure, weakness and harm-is partly due to a deep asymmetry of meaning: the occurrence of harmful outcomes clearly indicates the absence of safety, but the avoidance of harm does not necessarily indicate a state of safety (Reason, 2000). The fields of high reliability and resilience engineering emerged in large part to address this asymmetry by attempting to characterise and explain the positive characteristics of organisational activity that underpin safe, reliable and resilient performance.

Work in the field of resilience engineering heavily emphasises the need to understand and characterise the positive features of organisational safety-both in theory and in practice. A key tenet is the importance of examining why actions achieve their intended purposes and 'go right', rather than only paying attention 
to when things 'go wrong' (Hollnagel, 2013). The focus of resilience engineers is on the everyday performances that underpin success and safe outcomes, despite the complications, interruptions and uncertainties inherent to organisational life. Methodologically, there is an emphasis on focusing on organisational strengths and assets, and engaging in appreciative enquiry to understand what works well (Hollnagel, Braithwaite and Wears, 2013; Bushe, 2011). This focus on the positive features of organisational safety shares much with recent interest in 'positive deviance' that aims to identify unusual or exceptional success in order to understand and spread what works (Lawton et al, 2014; Bradley et al, 2009). The practical goal is to create situations in which 'as many things as possible go right' (Hollnagel, Wears and Braithwaite, 2015).

This emphasis on 'what works' in practice, and on positive features of organisational strength in the face of disruption and uncertainty, has equally defined much of the research that theorises processes of high reliability organising (Roberts, 1990; Vogus, 2011). The field of high reliability represents a decadeslong commitment to explaining the positive organisational and practical characteristics of safety-although in theories of high reliability, these are always examined in relation to how organisations handle and address disruptions, anomalies and unexpected events. That is, explanations of high reliability organising typically focus on how practical strengths become organised around operational weaknesses. 
Our analysis of the key organising processes that underpin the systemic use of in situ simulation points to a much deeper and complex set of interconnections between the light and dark sides of organisational safety. Systemic in situ simulation represents a strategy of safety improvement that simultaneously seeks to enhance positive organisational characteristics whilst also addressing organisational weaknesses and deficiencies. This analysis indicates that these two processes may be much closer in form and function than previously considered, and may be inseparable in practice. Systemic in situ simulation provides a space that is directly targeted at understanding, rehearsing and amplifying the practical patterns of activity that allow normal work to produce successful outcomes: developing the skills, knowledge and resources to prepare for and work around the challenges inherent to organisational life. The process of simulating, debriefing and reflecting on practice creates a safe space to identify and strengthen the activities that produces success. Equally, systemic in situ simulation is also directly targeted at identifying and addressing weaknesses in systems and practices. Simulating events in actual organisational settings allows organisational weaknesses and system deficiencies to be surfaced and addressed.

The systemic use of in situ simulation might therefore be explained through two processes: one identifies failures and weaknesses, and allows those to be addressed and improved; the other identifies successes and strengths, and allows those to be embedded and spread. However, both of these processes involve a relative improvement: they involve replacing one way of organising work with a 
better way. For example, in the maternity emergency of shoulder dystocia, the 'Pringle tube' solution to hand positioning represents a model of one small element of practice that is superior to other approaches that are more likely to cause harm. Simulation is used to spread this knowledge, develop this skill and embed this into the practical repertoire across an entire unit. This necessarily involves a more appropriate and successful element of practice displacing less successful or weaker forms of practice. Whether this process involves spreading 'what works'-building on the positive-or is instead focused on identifying and addressing a 'weakness'-eradicating a source of failure-is a somewhat arbitrary distinction.

In practice, the process of spreading 'good' practice and addressing 'bad' practice can be hard to neatly separate. There is always a displacement of one set of practices with another. Addressing problems and weaknesses requires developing better, stronger practices. And identifying and spreading successful and effective practices requires replacing prior weaker practices. Strength and weakness, and success and failure, are inextricably linked: they are a duality, and one cannot exist without the other. Recognising this duality has implications for how we understand the relationship between resilience and high reliability. The social processes of organising around distinct and disruptive episodes of failure (as explained by theories of high reliability) may echo the ways that routine work is organised around the variations and fluctuations of normal operations (which is 
the concern of resilience engineering). Differences between may be ones of scale and degree, rather than kind.

\subsection{Integrating proaction and reaction}

A persistent debate in organisational safety is about time: when does safety happen? Do the key activities that support safety occur before some sort of disruptive and adverse event, or do they occur after it? These issues are often framed in terms of reactive and proactive approaches to safety management. Safety strategies either focus on reactively responding to and recovering from events once they have occurred, or they focus on proactively preparing for and preventing events in the first place (Cox and Tait, 1991; Hollnagel, 2012). This question of timing has implicitly shaped debates about resilience, safety and risk since the 1980 s and informs some of the most fundamental assumptions that underpin theories of resilience engineering and high reliability organising. In particular, these assumptions have focused on the temporal dimension of disruptions and events in the production of organisational resilience and high reliability: either as a trigger for recovery and adaptation, or as a target for prevention and reduction. Our analysis of the organising processes of in situ simulation points to how assumptions about the proactive and reactive modes of safety management might be reinterpreted, and how the fundamental role of 
disruption in theories of resilience engineering and high reliability organising might be recast to bring these two fields closer together.

The issue of timing was fundamental to early analyses of resilience as a safety strategy. Drawing on ecological theories and economic models, Wildavsky (1988) originally defined resilience as a safety strategy based on flexibility and adaptation in response to failures: an ongoing process of distributed and repeated trial and error learning. Resilience was posed as a direct response to the centralised and anticipatory strategy of attempting to predict, prepare and prevent future harmful events-activities which can lead to wasting resources preparing for events that never happened, or losing opportunities to develop new knowledge and insight through experimentation and failure (Wildavsky, 1988). The field of resilience engineering has radically expanded the definition of resilience and continues to develop and enlarge its scope. Early definitions retained the assumption that resilience happened in response to events, defining resilience as the degree to which a system can handle disruptions and variations that fall outside the existing operating model of a system (Woods, 2006).

Increasingly, however, the overarching boundaries of the field of resilience engineering have expanded to encompass all modes of proactive and reactive forms of organisational safety (Westrum, 2006, p56). The working definition of resilience has equally expanded its temporal reach, to "the intrinsic ability of a system to adjust its functioning before, during, or after changes and disturbances, 
so that it can sustain required operations under both expected and unexpected conditions" (Hollnagel, 2012, p. 7). This definition seeks to integrate the activities of proactive and reactive safety management. And, while work within the field of resilience engineering rarely acknowledges or draws on models of resilience developed outside the field (Bergstrom, van Winsen and Henriqson, 2015), this temporal expansion echoes moves in other work on resilience, such as in political science and crisis management. For example, Comfort, Boin and Demchack (2010, p. 9) similarly define resilience as "the capacity of a social system ... to proactively adapt to and recover from disturbances that are perceived within the system to fall outside the range of normal and expected disturbances”. Resilience is increasingly conceptualised as a combination of both proactive and reactive activities of adaptation and recovery, that takes place in relation to some form of disruption.

Theories of high reliability organising equally grapple with issues of timing. An argument often made of high reliability organisations is that they must learn without the use of trial and error, lest the first error be the last trial (Weick and Sutcliffe, 2001). Presumably, the assumption here is that these error events will be significant in terms of material impact, and therefore must be avoided. At the same time, it is commonly asserted that learning from errors and organising around disruptions are the defining features of HROs (Rochlin, 1989; Roberts, Rousseau and La Porte, 1994). The events these statements refer to are presumably small and contained-the mishaps, anomalies and near-misses that 
are common in organisational life. Roe and Schulman (2016) have refined the relationship of high reliability to triggering events, and distinguish between precursor resilience and recovery resilience. Precursor resilience represents the preparatory work that seeks to maintain the capacity to respond to adverse events if need be, and recovery resilience represents the capacity to react and respond after the onset of a significant disturbance. This distinction echoes Boin and van Eeten's (2013) conceptualisation of proactive and reactive modes of resilience.

Our analysis of in situ simulation has deep and fundamental implications regarding the relationship between disruptions and organisational safety. One obvious observation is that rehearsing and simulating potential emergency scenarios is a mechanism of proactively preparing to reactively recover from disturbances. Simulation maintains and develops the skills needed to handle emergencies if and when they occur. More importantly, using in situ simulation to probe systems, identify weaknesses and improve processes represents a process of adaptation that unfolds around artificial or synthesised disruptions. The problems identified in simulations do not have any adverse or material disruption on organisational activity-but they can result in material changes to organisational processes. As such, in situ simulation demonstrates how adaptive organisational responses can be entirely uncoupled from materially disruptive events. In situ simulation creates a stream of symbolic or imagined disruptions that can be used to proactively rehearse reactive emergency responses, and that can also be used to 
adaptively reorganise work systems and redesign organisational processes-before any materially adverse event occurs.

Acknowledging this distinction between symbolically constructed disruptions (in simulated scenarios) and materially impactful disruptions (in actual service provision) shifts the reference point of safety away from materially disruptive events to culturally disruptive events. In doing so, it points to an important point of interconnection between theories of resilience engineering and high reliability (Macrae, 2014). Simulations are symbolic acts carried out in practice: put another way, simulation is a form of practical imagination. In situ simulation creates a symbolic arena in which to explore, uncover, adapt and improve real-world organisational systems and practices. Adaptations and improvements can be generated from events that are disruptive only in the cultural sense: they challenge common assumptions about how systems work, reveal collective blindspots in understanding and uncover gaps in shared practice (Turner, 1978).

Understood in this way, from a resilience engineering perspective in situ simulation is a way of continually testing work-as-imagined by comparing it with work-as-done, and actively generating and inquiring into surprising gaps in work systems (Hollnagel and Woods, 2006)-but in an entirely safe and protected space, where the only impacts of failure will be symbolic. From the perspective of high reliability organising, in situ simulation is a process that can actively produce surprising events that can continually challenge expectations, triggering processes 
of collective inquiry and adaptation (Rochlin, 1989; Weick, Sutcliffe and Obstfeld, 1999). In situ simulation allows organisations to explore and update the 'non-goal' that they are seeking to avoid (Roberts and Creed, 1993). How organisations generate, identify and respond to symbolic disruptions may therefore prove to be a productive area of intersection in future theories of high reliability and resilience engineering, and could help shift debates beyond temporal categories of proactive and reactive safety management.

A related challenge fundamental to safety management is dealing with entirely novel or completely unexpected events-circumstances that have never been experienced before and are entirely outside the range of current expectations and predictions. By definition, these 'fundamental surprises' (Lanir, 1986;

Christianson et al 2009) are unpredictable and inconceivable: they represent 'impossible' events (Wagenaar and Groeneweg, 1987)-at least from the perspective of those involved. This is one area where simulation would appear to meets its limits. Events that cannot be predicted or even imagined cannot be simulated. However, while it is not possible to imagine and rehearse every possible future event, the use of in situ simulation allows organisations to develop and stabilize a set of cognitive, social and material resources that can be drawn on and recombined when confronted with fundamental surprise. To an extent, every obstetric emergency event is novel and new, and differs from previous experiences in terms of the staff involved, the history of the patient and the resources to hand. In situ simulation allows the development of a repertoire of skills, knowledge, 
heuristics and recipes-while at the same time providing experience in applying and recombining those as needed in practice. It is still possible to proactively prepare for events that might only make sense in retrospect.

\subsection{Organising stability and change}

One of the most fundamental issues in understanding and explaining organisational safety is the deep tension between organisational stability and change: how organisations can handle variations and disruptions whilst delivering stable and reliable services (Wears, 2015). The fields of resilience engineering and high reliability organising define and address these issues in different ways. The key concepts and mechanisms in studies of high reliability organising seek to understand how experts and knowledge can be mobilised around unexpected events. The emphasis has been on the social and cognitive infrastructure that supports the ongoing monitoring and detection of deviations from normal operations (Weick and Roberts, 1993; Schulman, 1993), and flexible organising around key moments of disruption (Reason, 1997; Weick, Sutcliffe and Obstfeld, 1999). In resilience engineering, fluctuation and variability are viewed as deeply inherent to all practical work, and organisational safety is a result of continual adaptations, adjustments and flexible responses to the fluctuations in 
organisational life and the unpredictability inherent in any work setting (Hollnagel, 2012; Hollnagel, Braithwaite and Wears, 2013).

Theories of resilience engineering and high reliability organising emphasise the importance of learning and positive change over time: making sustained alterations and improvements to organisational systems. However, how these long-term changes are initiated and unfold, and how they relate to real-time responses to fluctuations and disruptions, remain relatively under-explored. Efforts to understand these tensions between stability and change have a long history in organisational studies-particularly in terms of the relationship between, on the one hand, the habits (Simon, 1976; Vogus and Hilligoss, 2016), plans (Suchman, 1987) and routines (Nelson and Winter, 1982) that underpin persistent and largely automatic patterns of organisational activity, and on the other hand, adaptive and consciously directed activities of change and improvement (Miettenen and Virkkunen, 2005).

Our analysis of systemic in situ simulation points to two fundamental modes of organising that help explain how organisational activities can simultaneously support both stability and change in the pursuit of organisational safety, and that also might offer a more integrated approach to understanding core mechanisms of organisational resilience and high reliability. One set of processes represents the accommodative, transient and temporary adjustments that are continually made moment-by-moment to catch, contain or compensate for operational disruptions 
and fluctuations. This is the ongoing repair, recovery and reaction that is continually needed to adjust to the fluctuations and variations in any organisational setting. The other set of processes represents the reflective, planned and effortful adaptations that are coordinated over time to improve systems and practices in light of disruptions. These are the punctuated moments of reform, reorganisation and redesign that are required to adapt to the disruptions and surprises that emerge in organisational activity. Both of these two complementary modes of organising are supported by systemic in situ simulation, and both of them appear to explain some of the most fundamental mechanisms underlying organisational resilience and high reliability. The former can be termed dynamic adjustment, following Weick (1987) and Woods (2006), and the latter can be termed adaptive reorganisation, following Hutchins (1996).

\subsubsection{Dynamic adjustment}

Dynamic adjustment represents the moment-by-moment adjustments, accommodations and responses that are required in practical work to handle the fluctuations and variations of normal organisational life. These adjustments may required considerable shifts in the way tasks are being performed or teams are being organised-for example, at the onset of a maternity emergency an immediate cascade of dynamic adjustments must be made to all ongoing activities 
and work practices, in order to organise tasks and attention around the emerging crisis. Alternatively, these dynamic adjustments may involve smaller scale adjustments to ongoing work, such as the adjustments that must occur if, for instance, the rate of blood loss during an emergency increases, or if a suspected diagnosis shifts from a typical bleeding situation to a more challenging situation of disseminated intravascular coagulation (DIC) that requires different tasks, routines and actions to be performed. Dynamic adjustment therefore spans the range of responsive and flexible activities that are characterised by theories of high reliability and resilience engineering: it encompasses the moments when significant groups of experts may self-organise around difficult operational problems (Rochlin, 1999; Weick, Sutcliffe and Obstfeld, 1999), and it encompasses the practical shifts and compensating actions that continually occur in the face of unpredictable variations (Wears and Vincent, 2013).

Systemic in situ simulation can create the organisational resources that support these sorts of ongoing dynamic adjustments, and it can embed the interactive patterns, social norms and cultural premises (Weick, 1987) that bound the limits of warranted and appropriate behaviour in different settings-what Hollnagel calls the "dynamic stability" (Hollnagel, 2006, p. 16) of practice. These organisational resources are cognitive and social as much as they are technical and material. They include the knowledge and skills required to detect signals of emerging problems. They include the shared expectations, assumptions and norms that underpin patterns of coordinated working. They include the trust, 
confidence and willingness to respond to surprising and novel situations. They include the algorithms, plans and protocols that support collective cognition. And they include the material infrastructure of emergency boxes and organisational systems that deliver equipment and support when it is needed.

These organisational resources are all required to support the ongoing dynamic adjustments required to deal with disruptive events, disturbances and surprises. Organisations remember by doing (Nelson and Winter, 1982): routines, skills and capabilities for acting are maintained by performing them. In situ simulation allows rehearsal and practice at deploying these resources to different types of challenging scenario, building a collective propensity to attend to certain cues, communicate in certain patterns and act in certain ways when confronted with unexpected events. Simulation provides the space in which organisational memory can be maintained and continually refreshed, even with regard to events that happen rarely and can occur in unique and surprising ways.

However, these plans and propensities for acting in certain ways should not be confused with the actual actions that are required when organisations confront risk. Reacting to challenging situations is always an inventive process that depends on in-the-moment judgement, skill and ingenuity, and is context dependent and contingent on the resources and materials at hand. Action should not be confused with the plans and preparations that go before it (Suchman, 1987; Macrae 2014). Organisational safety is always produced in the moment, by 
bringing together resources from past moments of socialisation (plans, recipes, protocols, routines, collective norms and shared assumptions) and combining those with the materials at hand at a specific moment in time (situated awareness, medical technologies, professional judgement and deployable knowledge). Dynamic adjustment is the practical work of drawing on and applying prior plans, propensities and preparation in order to act effectively in the moment.

\subsubsection{Adaptive reorganisation}

Adaptive reorganisation represents the effortful processes of reflection, invention and adaption that are required to reorganise and redesign practical work and organisational systems in light of disruptive and unexpected events. Adaptive reorganisation can involve the large-scale reorganisation of work practices, tools and support systems, such as developing a new set of more streamlined processes to request and deliver blood products to a maternity unit during an emergency. It can also involve much smaller, incremental improvements that are targeted at a focused area of practice, such as modifying or updating a protocol, or instituting a routine of hanging a sign on the door during team debriefings that indicates who on the ward has the keys to the drug cabinet. Adaptive reorganisation involves reflective, experimental and purposive change of the resources and systems that support organisational activities. It represents the longer-term process of learning 
and adaptation that occur within organisations in response to past experience and new evidence of emerging risks. These processes of long-term change and learning are often emphasised in the fields of resilience engineering and high reliability organising, through remain under-theorised (Weick, Sutcliffe and Obstfeld, 1999).

Systemic in situ simulation can provide a space to identify problems, examine systems, reflect on practices and test improvements. Critically reflecting on practices and systems is an essential feature of organisational adaptation and learning. In situ simulation allows different elements of collective practices and organisational systems to be turned into objects of inquiry themselves (Miettinen and Virkunnen, 2005). This allows standardised routines, tools and habits to be regularly tested, and reorganised where necessary, ensuring that even highly routinised practices and habits can remain intelligent (Miettinen, 2000) and flexible. In situ simulation can also ensure that the representations of work-asimagined-such as policies, guidelines, protocols and standards-can be routinely tested against the practical reality of work-as-done (Woods, 2006; Hollnagel, Wears and Braithwaite, 2015). As such, systemic in situ simulation creates an affordance for the generative work of producing knowledge about practice itself (Cook and Brown, 1999), as well as creating a space for adaptively reorganizing those practices. Symbolically disruptive events can act as the trigger for processes that reshape both organizational practices, and organizational representations of those practices, acting as a source of long term improvement and learning (Wenger, 1998; Macrae 2014). 


\section{Towards reliable resilience and resilient reliability}

The founding principles of the fields of resilience engineering and high reliability organising share much in common. Both aim to understand how organisations can maintain high levels of safety through adaptive and flexible work processes. Both are committed to understanding the positive characteristics and practical strengths that underpin organisational safety. And both are focused on understanding the practical and situated work of organisational life. Examining key practical strategies of safety improvement, such as in situ simulation, through these two complementary lenses provides one route to exploring some of the more fundamental issues in organisational safety. In safety management, as in research, getting closer to practice can reveal more about our theories: where they are useful, where they breakdown, where they connect and where they can be improved. This analysis of in situ simulation indicates a variety of ways that our understanding of organisational safety might be developed and refined. In particular, it suggests that more closely integrating ideas of resilience and reliability might be move us beyond overly simplistic dichotomies of success and failure, strength and weakness, stability and change (Wears, 2015).

Understanding organisational safety as a complex duality, defined by trade-offs, tensions and unexpected interactions, may offer new ways of integrating key 
elements of resilience engineering and high reliability organising, whilst also revealing the richness, subtlety and power in the practical work that creates organisational safety. 
Acknowledgements: We thank the Health Foundation, an independent charity working continuously to improve the quality of health care in the UK, for supporting this work through the authors' Improvement Science Fellowships. 


\section{References}

Arora, S., Hull, L., Fitzpatrick, M., Sevdalis, N. and Birnbach, D.J. (2015). Crisis

Management on Surgical Wards A Simulation-based Approach to Enhancing

Technical, Teamwork, and Patient Interaction Skills, Annals of Surgery, 261, 888893.

Ayres-de-Campos, D., Deering, S., and Siassakos, D. (2011). Sustaining simulation training programmes--experience from maternity care. BJOG: an International Journal of Obstetrics \& Gynaecology, 118(3), 22-26.

Batalden, P. 2010. Make the most of your turn. Lessons Learned in changing healthcare... and how we learned them November 2010: 179-192. Longwoods.

Bergström, J., van Winsen, R., \& Henriqson, E. (2015). On the rationale of resilience in the domain of safety: A literature review. Reliability Engineering \& Systems Engineering, 141, 131-141.

Bigley, G. A., and Roberts, K. H. (2001). The incident command system: High-reliability organizing for complex and volatile task environments. Academy of Management Journal, 44(6), 1281-1299. 
Boin, A. and Van Eeten, M. J. G. (2013). The resilient organization: A critical appraisal. Public Management Review, 15(3), 429-445.

Bradley, E. H., Curry, L. A., Ramanadhan, S., Rowe, L., Nembhard, I. M., \& Krumholz, H. M. (2009). Research in action: using positive deviance to improve quality of health care. Implementation Science, 4(1), 25-11.

Bushe, G.R. (2011) Appreciative inquiry: Theory and critique. In Boje, D., Burnes, B. and Hassard, J. (eds.) The Routledge Companion To Organizational Change. Oxford: Routledge.

Comfort, L. K., Boin, A. and Demchak, C. C. (2010). Designing Resilience: Preparing for Extreme Events. Pittsburgh: University of Pittsburgh Press.

Cook, S., and Brown, J. S. (1999). Bridging epistemologies: The generative dance between organizational knowledge and organizational knowing. Organization Science, 10(4), 381-400.

Cornthwaite, K., Crofts, J., Draycott, T., Siassakos, D. and Winter, C. (2015). Training for obstetric emergencies: PROMPT and shoulder dystocia. London: The Health Foundation. 
Cox, S. and Tait, N. R. S. (1991). Reliability, Safety and Risk Management: An Integrated Approach. London: Butterworth-Heinemann.

Crofts, JF, Bartlett, C, Ellis, D, Hunt, L.P. Fox, R and Draycott, T.J. (2006). Training for shoulder dystocia: a trial of simulation using low-fidelity and highfidelity mannequins. Obstetrics and Gynecology, 108(6), 1477-1485.

Dekker, S. W. A., Dahlström, N., van Winsen, R., \& Nyce, J. M. (2008). Crew resilience and simulator training in aviation. In: E. Hollnagel, C. Nemeth, \& S. W. A. Dekker (Eds.). Resilience Engineering Perspectives: Remaining sensitive to the possibility of failure, pp. 119-126. Aldershot, UK: Ashgate Publishing Co.

Draycott, T. (2013). Practical Obstetric Multi-Professional Training - PROMPT. London: The Health Foundation.

Draycott, T., Broad, G. and Chidley, K. (2000). The development of an eclampsia box and "fire drill". British Journal of Midwifery, 8(1), 26-30.

Draycott, T. J., Collins, K. J., Crofts, J. F., Siassakos, D., Winter, C., Weiner, C. P., \& Donald, F. (2015). Myths and realities of training in obstetric emergencies. Best Practice \& Research Clinical Obstetrics \& Gynaecology, 29(8):1067-76. 
Draycott, T. J., Crofts, J. F., Ash, J. P., Wilson, L. V., Yard, E., Sibanda, T., \& Whitelaw, A. (2008). Improving neonatal outcome through practical shoulder dystocia training. Obstetrics \& Gynecology, 112(1), 14.

Draycott, T., Sibanda, T., Owen, L., Akande, V., Winter, C., Reading, S., \& Whitelaw, A. (2006). Does training in obstetric emergencies improve neonatal outcome? BJOG: an International Journal of Obstetrics \& Gynaecology, 113(2), 177-182.

Driver, J. E., Gaba, D. M., and Lighthall, G. K. (2011). The benefits of using simulation in risk management and patient safety. In B. Youngberg (Ed.), Principles of Risk Management and Patient Safety. London: Jones \& Bartlett Publishers.

Dutta S, Gaba D, Krummel TM. (2006). To Simulate or Not to Simulate: What Is the Question? Annals of Surgery, 243(3), 301-303.

Fent, G., Blythe, J., Farooq, O. and Purva, M. (2015). In situ simulation as a tool for patient safety: a systematic review identifying how it is used and its effectiveness. BMJ Simulation and Technology Enhanced Learning, 1, 103-110.

Gaba, D. (2004). The future vision of simulation in health care. Quality and Safety in Health Care, 13(1), i2-i10. 
Gaba, D. M. (2010). Crisis resource management and teamwork training in anaesthesia. British Journal of Anaesthesia, 105(1), 3-6.

Gaba, D. M., Howard, S. K., Fish, K. J., Smith, B. E., and Sowb, Y. A. (2001). Simulation-Based Training in Anesthesia Crisis Resource Management (ACRM): A Decade of Experience. Simulation \& Gaming, 32(2), 175-193.

Gaba, D.G., Fish, K.J., Howard, S.K. and Burden, A.R. (2015). Crisis management in anaesthesiology (2nd ed). London: Elsevier Saunders.

Gallagher, C.J. and Corrado, T. (2014). The use of humour to enrich the simulation environment. In, The Comprehensive Textbook of healthcare simulation. Levine, A.I., DeMaria, S., Schwarz, A.D. and Sim, A.J. London: Springer.

Goldhaber-Fiebert, S. N., \& Howard, S. K. (2013). Implementing Emergency Manuals. Anesthesia \& Analgesia, 117(5), 1149-1161.

Goldhaber-Fiebert, S. N., Pollock, J., Howard, S. K., \& Bereknyei Merrell, S. (2016). Emergency manual uses during actual critical events and changes in safety culture from the perspective of anesthesia residents: a pilot study. Anesthesia and Analgesia, 123(3), 641-649. 
Haji, F A, Cheung, J J H, Woods, N, Regehr, G, de Ribaupierre, S, and Dubrowski, A. (2016). Thrive or overload? The effect of task complexity on novices' simulation-based learning. Medical Education, 50(9), 955-968.

Hamman, W. R., Beaudin-Seiler, B. M., Beaubien, J. M., Gullickson, A. M., Gross, A. C., Orizondo-Korotko, K., et al. (2009). Using in situ simulation to identify and resolve latent environmental threats to patient safety: case study involving a labor and delivery ward. Journal of Patient Safety, 5(3), 184-187.

Hollnagel, E. (2012). Proactive approaches to safety management. London: The Health Foundation.

Hollnagel, E. (2013). Making health care resilient: from safety I to safety II. In, Resilient Healthcare, Hollnagel, E, Braithwaite, J. and Wears, RL (Eds). Aldershot: Ashgate.

Hollnagel, E. and Woods, D. (2006). Epilogue: Resilience engineering precepts. In Resilience Engineering: Concepts and Precepts, Hollnagel, E., Woods, D.D. and Leveson, N. (Eds). Aldershot: Ashgate. 
Hollnagel, E., Braithwaite, J. and Wears, RL. (2013). How to make health care resilience. In, Resilient Healthcare, Hollnagel, E, Braithwaite, J. and Wears, RL (Eds). Aldershot: Ashgate.

Hollnagel, E., Paries, J., Woods, D. and Wreathall, J. (2012). Resilience Engineering in Practice: A Guidebook. Aldershot: Ashgate.

Hollnagel, E., Wears, RL. and Braithwaite, J. (2015). From Safety-I to Safety-II: A White Paper. World Health Organisation.

Hutchins, E. (1996). Cognition in the Wild. London: MIT Press.

Kingdon C., Neilson J, Singleton V, Gyte, G, Hart A, Gabbay, M, and Lavender, T. (2009). Choice and birth method: mixed-method study of caesarean delivery for maternal request. BJOG: An International Journal of Obstetrics and Gynaecology, 116(7):886-95.

Kirkup, B. (2015). The Report of the Morecambe Bay Investigation. The Report of the Morecambe Bay Investigation. London.

La Porte, T. R. and Consolini, P. (1991). Working in practice but not in theory: Theoretical challenges of high reliability organizations. Journal of Public Administration Research and Theory, 1 (1), 19-47. 
La Porte, T.R. (1994). A strawman speaks up: Comments on The Limits of Safety. Journal of Contingencies and Crisis Management, 2, 207-211.

Landsdalen H, Berge M, Brattebø G. (2009). Making simulations more real: experiences from an advanced resuscitation provider course. Scandinavian Journal of Trauma, Resuscitation and Emergency Medicine, 17(3):O20.

Lawton, R., Taylor, N., Clay-Williams, R., \& Braithwaite, J. (2014). Positive deviance: a different approach to achieving patient safety. BMJ Quality and Safety, 23(11), 880-883.

MacKenzie, I., Shah, M., Lean, K., Dutton, S., Newdick, H. and Tucker, D. E. (2007). Management of shoulder dystocia: trends in incidence and maternal and neonatal morbidity. Obstetrics and Gynecology. 110(5), 1059-1068.

Macrae, C. (2010). Regulating resilience? Regulatory work in high-risk arenas. In Hutter, B. (Ed), Anticipating Risks and Organising Risk Regulation. Cambridge: Cambridge University Press.

Macrae, C. (2013). Reconciling regulation and resilience. In Hollnagel, E., Braithwaite, J., and Wears, R. (Eds), Resilient Health Care. Aldershot: Ashgate. 
Macrae, C. (2014). Close Calls: Managing Risk and Resilience in Airline Flight Safety. London: Palgrave Macmillan.

Marshall, S. (2013). The Use of Cognitive Aids During Emergencies in Anesthesia. Anesthesia \& Analgesia, 117(5), 1162-1171.

MCHRC. (2000). Confidential Enquiry into Stillbirths and Deaths in Infancy. 7th annual report. London: Maternal and Child Health Research Consortium.

Miettinen, R. (2000) 'The Concept of Experiential Learning and John Dewey's Theory of Reflective Thought and Action', International Journal of Lifelong Education 20(1): 54-72.

Miettinen, R. and Virkkunen, J. (2005). Epistemic Objects, Artefacts and Organizational Change. Organization, 12(3), 437-456.

Nelson, R. and Winter, S. (1982) An Evolutionary Theory of Economic Change. Cambridge, MA: Belknap Press.

NHSLA. (2000). CNST standards: Maternity manual. London: NHS Litigation Authority. 
NHSLA. (2012). Ten years of maternity claims: an analysis of NHS Litigation Authority data. London: NHS Litigation Authority.

Nielsen PE, Goldman MB, Mann S, Shapiro DE, Marcus RG, Pratt SD, et al. Effects of teamwork training on adverse outcomes and process of care in labor and delivery: a randomized controlled trial. Obstetrics and Gynecology, 2007, 109, $48-55$.

Nielsen PE, Goldman MB, Mann S, Shapiro DE, Marcus RG and Pratt SD. (2007). Effects of teamwork training on adverse outcomes and process of care in labor and delivery: a randomized controlled trial. Obstetrics and Gynaecology, 109, 48-55.

Patterson, M. D., Geis, G. L., Falcone, R. A., LeMaster, T., \& Wears, R. L. (2013). In situ simulation: detection of safety threats and teamwork training in a high risk emergency department. BMJ Quality and Safety, 22(6), 468-477.

Patterson, M., Kessler, D.O. and Auerbach, M. (2015). The use of in situ simulation to detect latent safety threats in paediatrics: a cross-sectional survey. BMJ Simulation and Technology Enhanced Learning, 1, 77-82.

RCM. (2002). Clinical risk management, Paper 2: Shoulder dystocia. London: Royal College of Midwives. 
RCOG. (1999) Towards safer childbirth: minimum standards for the organisation of labour wards. London: Royal College of Obstetricians and Gynaecologists.

RCOG. (2009). Postpartum Haemorrhage, Prevention and Management (Greentop Guideline No. 52). London: Royal College of Obstetricians and Gynaecologists.

Reason, J. (2000). Safety paradoxes and safety culture. Injury Control and Safety Promotion, 7(1), 3-14.

Resar, R. K. (2006). Making Noncatastrophic Health Care Processes Reliable: Learning to Walk before Running in Creating High-Reliability Organizations. Health Services Research, 41(4/2), 1677-1689.

Riley, W., Davis, S., Miller, K. M., Hansen, H., and Sweet, R. M. (2010). Detecting breaches in defensive barriers using in situ simulation for obstetric emergencies. Quality \& Safety in Health Care, 19(3), i53-6.

Riley, W., Lownik, E., Parrotta, C., RN, K. M., and Davis, S. (2011). Creating High Reliability Teams in Healthcare through In situ Simulation Training. Administrative Sciences, 1(1), 14-31.

Roberts, K. (1990). Some characteristics of one type of high reliability organisation. Organization Science, 1 (2), 160-176. 
Roberts, K. H. and Creed, W. E. D. (1993). Epilogue. In K. H. Roberts (ed.), New Challenges to Understanding Organizations. New York: Macmillan.

Roberts, K. H., Rousseau, D. M. and La Porte, T. R. (1994). The culture of highreliability: Quantitative and qualitative assessment aboard nuclear-powered aircraft carriers. The Journal of High Technology Management Research, 5 (1), $141-161$.

Rochlin, G. I. (1989). Informal organizational networking as a crisis-avoidance strategy: US Naval flight operations as a case study. Industrial Crisis Quarterly, 3, 159-176.

Rochlin, G.I. (1999). Safe operation as a social construct, Ergonomics, 42(11), 1549-1560.

Roe, E. and Schulman, P. (2016). Reliability and Risk: The Challenge of Managing Interconnected Infrastructures. Stanford: Stanford University Press.

Rosen, K. (2014). The history of simulation. In. The Comprehensive Textbook of Healthcare Simulation. Adam I. Levine, Samuel DeMaria Jr., Andrew D Schwartz, Alan J. Sim. (Eds). London: Springer. 
Sagar, R., Draycott, T., \& Hogg, S. (2015). The role of insurers in maternity safety. Best Practice \& Research Clinical Obstetrics \& Gynaecology, 29(8), 1126-1131.

Schmidt, E., Goldhaber-Fiebert, S. N., Ho, L. A., and McDonald, K. M. (2013). Simulation Exercises as a Patient Safety Strategy. Annals of Internal Medicine, $158(2), 426$.

Schulman, P. R. (1993). The negotiated order of organisational high reliabilty. Administration and Society, 25(3), 353-372.

Schulman, P. R. (2004). General attributes of safe organisations. Quality \& Safety in Health Care, 13(2), ii39-ii44.

Siassakos, D., Bristowe, K., Draycott, T. J., Angouri, J., Hambly, H., and Winter, C. (2011). Clinical efficiency in a simulated emergency and relationship to team behaviours: a multisite cross-sectional study. BJOG: an International Journal of Obstetrics \& Gynaecology, 118(5), 596-607.

Siassakos, D., Crofts, J., Winter, C., Weiner, C., and Draycott, T. (2009). The active components of effective training in obstetric emergencies. BJOG: an International Journal of Obstetrics \& Gynaecology, 116(8), 1028-1032. 
Siassakos, D., Fox, R., Bristowe, K., Angouri, J., Hambly, H., Robson, L., and Draycott, T. J. (2013). What makes maternity teams effective and safe? Lessons from a series of research on teamwork, leadership and team training. Acta Obstetricia Et Gynecologica Scandinavica, 92(11), 1239-1243.

Siassakos, D,, Fox, R,. Hunt, L., Farey, J., Laxton, C., Winter, C., and Draycott, T. (2011). Attitudes toward safety and teamwork in a maternity unit with embedded team training. American Journal of Medical Quality, 26(2), 132-137.

Siassakos, D., Hasafa, Z., Sibanda, T., Fox, R., Donald, F., Winter, C., and Draycott, T. (2009). Retrospective cohort study of diagnosis-delivery interval with umbilical cord prolapse: the effect of team training. BJOG: an International Journal of Obstetrics \& Gynaecology, 116(8), 1089-1096.

Simon, H. A. (1976) Administrative Behavior. A Study of Decision-making Process in Administrative Organization, 3rd edn. London: Free Press.

Suchman, L. 1987. Plans and situation actions: The Problem of Human-Machine Communication. Cambridge.

Sutcliffe, K. M., and Weick, K. E. (2003). Hospitals as cultures of entrapment: a re-analysis of the Bristol Royal Infirmary. California Management Review, 45(2), $73-84$ 
Tan, S.S. and Sarker, S.K. (2011). Simulation in surgery: a review. Scottish Medical Journal, 56(2), 104-9.

Thompson, S., Neal, S., and Clark, V. (2004). Clinical risk management in obstetrics: eclampsia drills. British Medical Journal, 328(7434), 269-271.

TJC. (2004). Sentinel event alert: Issue 30. Preventing infant death and injury during delivery. Oakbrook Terrace: The Joint Commission.

Turner, B. (1978). Man-Made Disasters. London: Wykeham.

Ventre, K. M., Barry, J. S., Davis, D., Baiamonte, V. L., Wentworth, A. C., and Pietras, M. (2014). Using in situ simulation to evaluate operational readiness of a children's hospital-based obstetrics unit. Simulation in Healthcare: the Journal of the Society for Simulation in Healthcare, 9(2), 102-111.

Vogus, T. J. (2011). Mindful Organizing: Establishing and Extending the Foundations of Highly Reliable Performance. In Cameron, K. and Spreitzer, G. M. (Eds.). The Oxford Handbook of Positive Organizational Scholarship: 664676. Oxford: Oxford University Press.

Vogus, T. J., and Hilligoss, B. (2016). The underappreciated role of habit in highly reliable healthcare. BMJ Quality and Safety, 25(3), 141-146. 
Wagenaar, W. A. and Groeneweg, J. (1987). Accidents at sea: Multiple causes and impossible consequences. International Journal of Man-Machine Studies, 27, $587-598$.

Walker, S. T., Sevdalis, N., McKay, A., Lambden, S., Gautama, S., Aggarwal, R., \& Vincent, C. (2013). Unannounced in situ simulations: integrating training and clinical practice. BMJ Quality and Safety, 22(6), 453-458.

Wears, R. L. (2015). Standardisation and Its Discontents. Cognition, Technology \& Work, 17(1), 89-94.

Wears, R.L. and Vincent, C. (2013). Relying on resilience: too much of a good thing? In, Resilient Healthcare, Hollnagel, E, Braithwaite, J. and Wears, RL (Eds). Aldershot: Ashgate.

Weick, K. E. (1987). Organizational culture as a source of high reliability. California Management Review, 29 (2), 112-127.

Weick, K. E. (1995). What theory is not, theorizing is. Administrative Science Quarterly, 40(3), 385-390.

Weick, K. E. and Roberts, K. H. (1993). Collective mind in organizations: Heedful interrelating on flight decks. Administrative Science Quarterly, 38, 357-381. 
Weick, K. E. and Sutcliffe, K. M. (2001). Managing the Unexpected: Assuring High Performance in an Age of Complexity. San Francisco: Jossey Bass.

Weick, K. E., Sutcliffe, K. M. and Obstfeld, D. (1999). Organizing for high reliability: Processes of collective mindfulness. Organizational Behaviour, 21, 81-123.

Weick, K. E., Sutcliffe, K. M., and Obstfeld, D. (1999). Organizing for high reliability: Processes of collective mindfulness. In R. S. Sutton and B. M. Staw (Eds.), Research in Organizational Behavior. Stanford: Jai Press.

Weinger, M. B., Burden, A. R., Steadman, R.H. and Gaba, D.M. (2014). This is not a test! Misconceptions surrounding the maintenance of certification in anesthesiology simulation course. Anesthesiology, 121(3), 655-659.

Wenger, E. (1999). Communities of Practice: Learning, Meaning, and Identity. Cambridge: Cambridge University Press.

Westrum, R. (2006). A typology of resilience situatinos. In, Resilience Engineering: Concepts and Precepts, Hollnagel, E., Woods, D.D. and Leveson, N. (Eds). Aldershot: Ashgate.

Wildavsky, A. (1988). Searching for Safety. London: Transaction. 
Winter, C., Crofts, J., Laxton, C., Barnfield, S. and Draycott, T. (2012b). PROMPT Trainer's Manual (2nd ed). London: RCOG Press.

Winter, C., Crofts, J., Laxton, C., Barnfield, S. and Draycott, T. (2012a). PROMPT Course Manual (2nd ed). London: RCOG Press.

Wisborg T, Brattebø G, Brattebø J, Brinchmann-Hansen A. (2006). Training multiprofessional trauma teams in Norwegian hospitals using simple and low cost local simulations. Education for Health, 19(1), 85-95.

Woods, D. (2006). Essential characteristics of resilience. In, Resilience Engineering: Concepts and Precepts, Hollnagel, E., Woods, D.D. and Leveson, N. (Eds). Aldershot: Ashgate. 